\title{
Multi-Modal Nano Particle Labeling of Neurons
}

\author{
Lilac Amirav $^{1 *}$, Shai Berlin²*, Shunit Olszakier1,2, Sandip K. Pahari1 and Itamar Kahn²* \\ ${ }^{1}$ Schulich Faculty of Chemistry, Technion - Israel Institute of Technology, Haifa, Israel, ${ }^{2}$ Department of Neuroscience, Ruth \\ and Bruce Rappaport Faculty of Medicine, Technion - Israel Institute of Technology, Haifa, Israel
}

OPEN ACCESS

Edited by:

loan Opris,

University of Miami, United States

Reviewed by:

Preston Snee,

University of lllinois at Chicago,

United States

Natalie Julie Serkova, University of Colorado Denver,

United States

Hari S. Sharma,

Uppsala University, Sweden

${ }^{*}$ Correspondence:

Lilac Amirav

lilac@technion.ac.il

Shai Berlin

shai.berlin@technion.ac.il

Itamar Kahn

kahn@technion.ac.il

Specialty section:

This article was submitted to

Neural Technology,

a section of the journal

Frontiers in Neuroscience

Received: 28 July 2018

Accepted: 08 January 2019

Published: 01 February 2019

Citation:

Amirav L, Berlin S, Olszakier S,

Pahari SK and Kahn I (2019)

Multi-Modal Nano Particle Labeling

of Neurons. Front. Neurosci. 13:12.

doi: 10.3389/fnins.2019.00012
The development of imaging methodologies for single cell measurements over extended timescales of up to weeks, in the intact animal, will depend on signal strength, stability, validity and specificity of labeling. Whereas light-microscopy can achieve these with genetically-encoded probes or dyes, this modality does not allow mesoscale imaging of entire intact tissues. Non-invasive imaging techniques, such as magnetic resonance imaging (MRI), outperform light microscopy in field of view and depth of imaging, but do not offer cellular resolution and specificity, suffer from low signal-to-noise ratio and, in some instances, low temporal resolution. In addition, the origins of the signals measured by MRI are either indirect to the process of interest or hard to validate. It is therefore highly warranted to find means to enhance MRI signals to allow increases in resolution and cellular-specificity. To this end, cell-selective bi-functional magnetofluorescent contrast agents can provide an elegant solution. Fluorescence provides means for identification of labeled cells and particles location after MRI acquisition, and it can be used to facilitate the design of cell-selective labeling of defined targets. Here we briefly review recent available designs of magneto-fluorescent markers and elaborate on key differences between them with respect to durability and relevant cellular highlighting approaches. We further focus on the potential of intracellular labeling and basic functional sensing MRI, with assays that enable imaging cells at microscopic and mesoscopic scales. Finally, we illustrate the qualities and limitations of the available imaging markers and discuss prospects for in vivo neural imaging and large-scale brain mapping.

Keywords: MRI, iron oxide nanoparticles, light microscopy, contrast agents, magneto fluorescence nanoparticle labeling

\section{INTRODUCTION}

A central goal in neuroscience research is the development of imaging methodologies for longitudinal single neuron interrogation in the intact animal. High-resolution light-microscopy (LM) imaging of neurons in vivo enables structural and functional mapping, as well as remote optical control with exquisite spatiotemporal resolution; shedding light on some of the most 
fundamental questions related to neural morphology and function in health and disease. However, LM offers but a glimpse of the brain in vivo, typically providing access to very small fieldsof-view and depths of the mammalian brain (Silva, 2017; Zong et al., 2017). Thus, non-invasive imaging modalities that can reveal the structure and function of neurons in the entire brain, i.e., at the mesoscale, could provide the complementary means needed for this ambitious endeavor. Magnetic resonance imaging (MRI) is particularly attractive to meet this goal, owing to its soft-tissue imaging capabilities, and the ability to image the entire organ non-invasively and repeatedly over a long period of time, through the intact skull and at any depth, with no known adverse impact on the tissue.

As commonly used, MRI provides an indirect measure of the brain's structure and function, owing to the manner by which signals are acquired - by perturbing the alignment of the highly abundant hydrogen atoms nuclear spins found in this tissue. In structural MRI, this signal is indistinguishable between the intra- versus extra-cellular environments and across cell types. Similarly, the contrast mechanism commonly measured by functional magnetic resonance imaging (fMRI) is also indirect (Logothetis, 2008; Kim and Ogawa, 2012). Intrinsic signals for fMRI arise from the ratio between oxygenated and deoxygenated hemoglobin, a mesoscopic metabolic measure, rather than measures of electrical activity or intracellular physiological events, such as $\mathrm{Ca}^{2+}$ concentration changes or neurotransmitter release. In recent years, several technological advances, including magnetic fields significantly higher than those commonly used in clinical settings, novel radio-frequency coils with a large number of densely-spaced small coil elements, and the use of unique dielectric materials, allow to indirectly track the activity of smaller and smaller populations of neurons in the brain (Ugurbil, 2016). Despite these innovations, the signals are still minute, of relatively poor signal-to-noise ratio (SNR) and indirect. To overcome these limitations, means to increase contrast of defined targets are under development, in particular MRI-compatible contrastagents that are designed to detect specific molecular targets in the brain (Mukherjee et al., 2017; Ghosh et al., 2018). As contrastagents are used routinely in clinical MRI scans, novel classes of contrast agents suitable for use in humans will certainly enhance and expand the capabilities of the technique. We propose that bi-functional materials that serve as hybrid contrast-agents for multiple imaging modalities at once, notably LM and MRI, will open new fronts for structural and functional whole-brain imaging at higher resolution and with target specificity.

The design and synthesis of bi-functional materials is an active research area with a significant impact on a wide range of technological applications (Corr et al., 2008; Gao et al., 2009; Suh et al., 2009; Qin and Bischof, 2012; Cheng et al., 2014). From an imaging point-of-view, combining several different properties onto a single agent can functionalize it towards many types of imaging and detection modalities; greatly extending its diagnostic, and potentially therapeutic, value. Indeed, emerging multifunctional contrast agents have been shown to label cancerous cells (Sailor and Park, 2012; Dawidczyk et al., 2014; Sanna et al., 2014) or genetically-modified cells in vivo (Kim et al., 2008; Muthu et al., 2014; Ortgies et al., 2016) for detection by MRI and validation by LM. Target selective imaging is a prerequisite for image-guided interventions (including surgery and ablation therapy) (Li, 2014). Beyond imaging, multifunctional contrast agents may also include pharmacological agents to concomitantly visualize and treat diseases in an all-in-one therapeutic and diagnostic (theranostic) approach (Yoo et al., 2011; Lim et al., 2015), for which there is a growing number of examples (Gao, 2018). Specifically, iron oxide nanoparticles are utilized for magneto-responsive therapy, where the responsiveness of the nanoparticles to an external magnetic field is used in order to increase the accumulation of the particles in a target tissue (magnetic targeting), or for exogenous physical stimuli release of cargo gene or drug molecules (Lee et al., 2015). However, this field is still in its infancy, with significant limitations in bona fide labeling of defined cellular targets and at obtaining sufficient particle accumulation at desired locations for gaining higher resolutions; notably to the single-cell level.

Cell-targeted contrast agents could provide the means to increase target-specificity and resolution by adhering and accumulating around or within cells. Extracellular contrast agents are typically aimed at reversibly binding proteins exposed to the extracellular-milieu. However, owing to proteinturn-over, limited expression of the target-protein, expression of some proteins in a large variety of cell types and limited membrane surface-area (Saka et al., 2014), such agents do not typically provide sufficient contrast of defined-cells; especially not for prolonged durations (Mukherjee et al., 2017). Intracellular contrast agents, on the other hand, may bypass several of these limitations and, thereby, potentially provide an extended imaging time-window. For instance, membrane expression levels and protein turn-over are less likely to impact the latter. In addition, the intracellular space (i.e., cytoplasm) greatly exceeds that of the membrane surface, allowing for the accumulation of larger amounts of contrast agents and, consequently, to provide higher signals. Intracellular accumulation also slows washout of the agent, thus extending the imaging periods, and it may also localize the activity of a therapeutic agent, or enable a weak drug (e.g., with a high median effective dose) to become efficient exclusively in the desired cellular population where the drug has been concentrated.

To meet their full potential, intracellular contrast-agents and their further development should benefit from better understanding of their cellular uptake mechanisms. Of particular interest is information on the modes of cellular uptake, their efficiency and kinetics, subcellular distribution of contrast agents following uptake, saturation concentrations, clearance and, notably, toxicity. Gaining control over these parameters will open the door towards novel basic and clinical applications.

A key requirement towards meeting this goal is the ability to track and validate the intracellular accumulation of contrast agents with high spatiotemporal resolution, significantly higher than what is currently afforded by MRI, and over an extended period of time. Here, multifunctional contrast agents that can be detected by both LM and MRI are particularly useful, lending themselves to achieve this task. Fluorescence imaging provides exquisite high-resolution means to explore and validate the 
different features of cellular uptake. It can be used to assess the accumulation of specific contrast agents at the cellular, subcellular, protein or even single molecule level, and at very high temporal resolution. To this end, several magneto-fluorescent hybrid systems are currently under development.

\section{MAGNETO FLUORESCENCE HYBRID NANOPARTICLES}

In order to combine LM and MRI, appropriate agents for both imaging modalities first need to be considered based on their signal strength, toxicity, stability and size. Several classes of MRI-compatible contrast agents are available (Geraldes and Laurent, 2009); with iron oxide nanoparticles meeting most of these requirements, namely provide a strong and stable MRI-signature, with little effects on cellular physiology (e.g., Uchiyama et al., 2015; and see review Shen et al., 2017). Consequently, iron oxide nanoparticles are commonly employed in the field. In particular, these have been rendered bi-modal by their conjugation to organic fluorescent dyes, such as fluorescein isothiocyanate, rhodamine B, and Cy5.5; all commonly employed bright markers for LM (Wysocki and Lavis, 2011). However, organic dyes may undergo rapid photobleaching and/or photochemical degradation (shortening the imaging time-window), or produce cytotoxic byproduct (e.g., reactive oxygen species; Zheng et al., 2014) and damage to the biological system under investigation. To address the instability of organic dyes, several reports describe encapsulation of dyes within silica to provide protection (Ow et al., 2005; Piao et al., 2008), but this provides only a moderate protection and comes at the cost of larger particles size. Quantum dots (QDs), inorganic fluorescent semiconductor nanoparticles, are an attractive alternative (Kim et al., 2004; Medintz et al., 2005). QDs have a high molar extinction coefficient and fluorescence quantum yield, broad absorption, and narrow tunable emission spectra, in addition to excellent temporal stability and resistance to photobleaching. These make QDs particularly advantageous over other fluorescent agents. However, coupling of QDs to MR contrast agents, specifically iron oxide nanoparticles, is not straightforward, and likely the reason why this has not been commonly achieved. This is mainly because a direct contact between the semiconductor and magnetic domain can lead to strong electronic coupling and strong attenuation of the QDs' fluorescence. Indeed, traditional heterodimer structures, namely placing the semiconductor QD directly on the surface of an iron oxide nanoparticle, are not sufficiently bright for optical imaging (Selvan et al., 2007). Fluorescence quenching can be minimized, or prevented entirely, if effective separation is achieved (Boldt et al., 2011;Bigall et al., 2012; Feld et al., 2015; Harris et al., 2016). Hence, alternative synthetic strategies for the fabrication of magneto-fluorescent materials include conjugation of separate nano-constructs, or co-encapsulation into organic structures or inorganic materials (Kim et al., 2006; Kim and Taton, 2007; Insin et al., 2008; Park et al., 2008; Roullier et al., 2008; Erogbogbo et al., 2010; Fan et al., 2010; Kas et al., 2010; Di Corato et al.,
2011; Shibu et al., 2013; Cho et al., 2014; Lee et al., 2015). These conceptual designs are expected to prevent undesirable interactions within the hybrid that could abrogate the respective properties.

Two prominent examples of such designs are presented in Figures 1a,b. Bawendi and co-workers (Chen et al., 2014) developed colloidal superstructures comprised of close-packed magnetic nanoparticle cores that are fully surrounded by a shell of fluorescent QDs, and the core-shell superparticle is coated with a protective silica shell. These super-nanoparticles exhibit high magnetic content (with $\mathrm{T}_{2}$ relaxivity of $402.7 \mathrm{mM}^{-1} \mathrm{~S}^{-1}$ ) along unperturbed fluorophore loading, with an overall diameter of $\sim 100 \mathrm{~nm}$. Weller and co-workers (Feld et al., 2015) used polystyrene to co-encapsulate iron oxide nanoparticles with quantum rods, thereby preserving the fluorescence and magnetism of the separate components, with particle diameters ranging from 74 to $150 \mathrm{~nm}$, and relaxivity of $164 \mathrm{mM}^{-1} \mathrm{~S}^{-1}$.

Recently, we presented a novel design strategy for the fabrication of ultra-small ( $\sim 15 \mathrm{~nm}$ hydrodynamic size) magnetofluorescent nanoparticles (Pahari et al., 2018). In lieu of conjugation or co-encapsulation of the separate magneto and fluorescent components into an insulating matrix, both were combined into a single entity with a unique morphology. The optically active semiconductor QD was encapsulated directly into a hollow paramagnetic iron oxide shell that serves as the MRI contrast agent (Figure 1c). Despite their small size, these nanoparticles provide contrast enhancement with a relaxivity of $304 \mathrm{mM}^{-1} \mathrm{~S}^{-1}$, making them comparable to the much bigger aforementioned systems, and superior to commercial contrast agents such as $\mathrm{Fe}_{3} \mathrm{O}_{4}$ nanoparticles (26.8 $\left.\mathrm{mM}^{-1} \mathrm{~S}^{-1}\right)$. This so-called "yolk-shell" morphology prevents the undesired interactions within the hybrid that would diminish the properties of the semiconductor and magnetic domains. Furthermore, this architecture offers a high level of tunability with respect to size, composition and surface functionalization. The photoluminescence quantum yield of the yolk-shell nanoparticles was typically $\sim 16 \%$ (at $450 \mathrm{~nm}$ excitation light), comparable to the $\sim 12 \%$ photoluminescence quantum yield (at $405 \mathrm{~nm}$ ) of the silica coated core-shell superparticles. This yield was sufficient for single particle tracking (Chen et al., 2014), and for optical microscopy imaging of nanoparticle clusters within single cells, or of 3D cell cultures (as seen in Figure 2; Pahari et al., 2018). The decrease in photoluminescence quantum yield compared with that of free QDs, that could be as high as 94\%, is attributed to the overlapping absorption with that of the iron oxide.

Noteworthy is the fact that for the morphologies presented in Figure 1, the fluorescent QDs may be altered with no effect on overall size, surface characteristics and magnetic properties of the particle. Hence, we anticipate that future hybrids will replace the Cd-based semiconductor with nontoxic and biocompatible alternatives and incorporate materials with efficient near-infrared (NIR) optical activity in both absorption and fluorescence; wavelengths more suitable for in vivo applications. This adjustment will also minimize the spectral overlap between the absorption of the core and that of the 

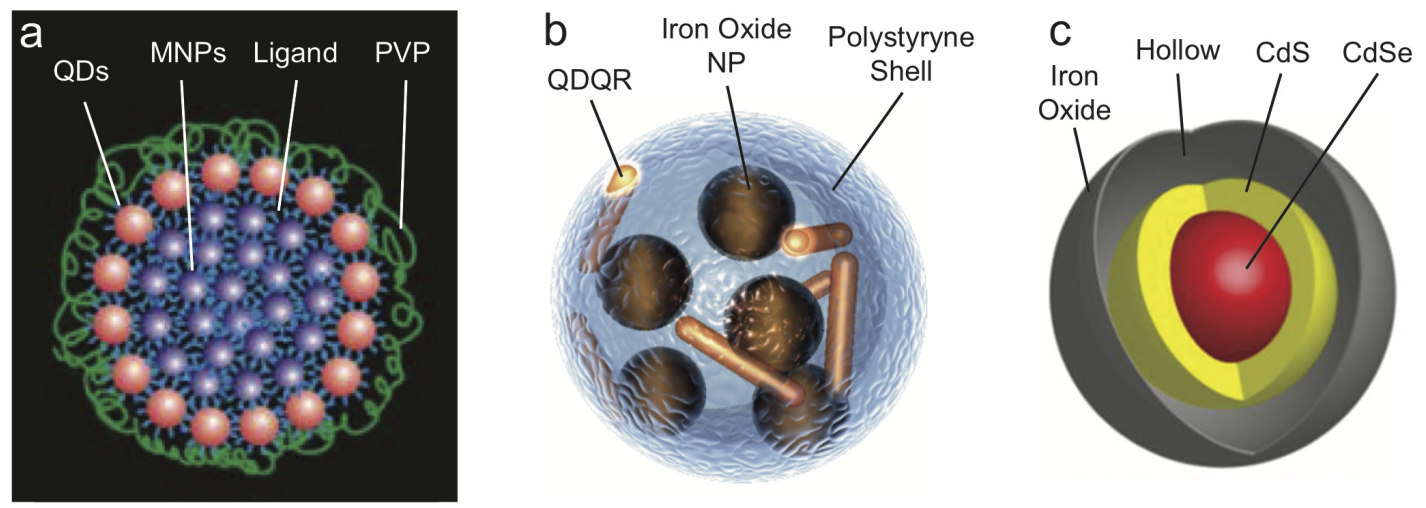

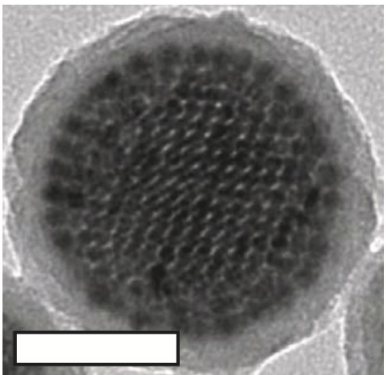

$50 \mathrm{~nm}$

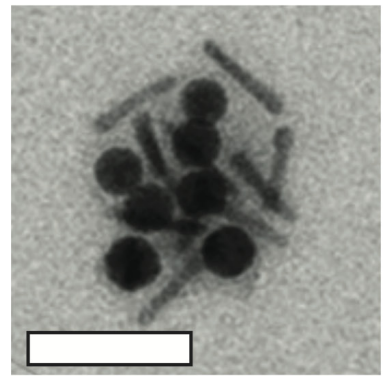

$50 \mathrm{~nm}$

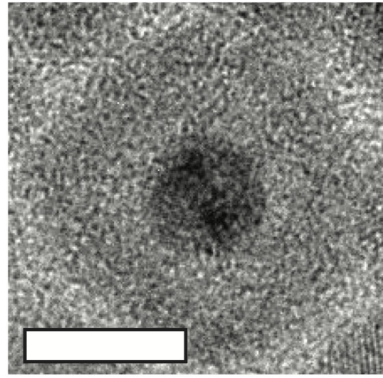

$5 \mathrm{~nm}$

FIGURE 1 | Magneto-Fluorescent Nanoparticles. (a) Schematic illustration (top) and TEM micrograph (bottom) of co-assembled super-nanoparticles consisting of closed-packed $\mathrm{Fe}_{3} \mathrm{O}_{4}$ magnetic nanoparticles "core" surrounded by a "shell" of fluorescent CdSe@CdS quantum dots (QDs). Following coating with silica, the superstructure obtain an average diameter of $100 \mathrm{~nm}$. Adapted with permission from Chen et al. (2014). Copyright (2014) Springer Nature. (b) Schematic illustration and TEM micrograph of magneto-fluorescent nanohybrid produced by two-step co-encapsulation of iron oxide nanoparticles and fluorescent CdSe@CdS quantum dots/quantum rods (QDQRs) into polystyrene shell, with a $50 \mathrm{~nm}$ diameter structures. Adapted with permission from Feld et al. (2015). Copyright (2015) John Wiley and Sons. (c) Schematic illustration and TEM micrograph of magneto-fluorescent yolk-shell hybrid structures composed of CdSe@CdS core that is encapsulated within a hollow- $\mathrm{Fe}_{2} \mathrm{O}_{3}$ shell, with a $10 \mathrm{~nm}$ diameter. Adapted with permission from Pahari et al. (2018). Copyright (2018) American Chemical Society.

hollow shell, and is expected to improve the photoluminescence quantum yield.

An interesting alternative for multimodal imaging is provided by incorporation of manganese ions into the $\mathrm{QD}$, hence offering the semiconductor itself paramagnetic characteristics (Sitbon et al., 2014). These structures may serve as T1-contrast agents, influencing the longitudinal relaxation time, whereas superparamagnetic iron oxide nanoparticles are employed as T2-contrast agents, influencing the transverse relaxation time.

\section{INTRACELLULAR ACCUMULATION OF NANO-PARTICLES}

Particle-size is a critical criterion that will determine the mechanism by which it will be internalized into the cell, with major influences on cellular uptake efficiency and kinetics, and subcellular distribution (He et al., 2010; Huang et al., 2010; Verma and Stellacci, 2010; Shang et al., 2014; Zhang et al., 2015). Note that this refers to the overall size of the structure, including the inorganic and organic shells. The modes of internalization can vary quite extensively, ranging from processes such as opsonization and phagocytosis (Gustafson et al., 2015), through clathrin/caveolar-mediated endocytosis (Hachani et al., 2017) and receptor-mediated endocytosis (Qiao et al., 2012), to macroand pinocytosis. The size of a contrast agent will also impact the means by which it escapes from the endosomal/lysosomal compartments and enters the cytoplasm (for a review see, Oh and Park, 2014).

A significant factor for in vivo imaging is the effect of the hydrodynamic diameter of the contrast agent on its resident circulation time (Kievit and Zhang, 2011). Nanoparticles with a hydrodynamic diameter of less than $10 \mathrm{~nm}$, i.e., smaller than the pore size of the glomerulus, are subjected to rapid excretion and thus have relatively short dwell-time in the blood stream (Chapman et al., 1999; Olmsted et al., 2001; Choi et al., 2007; Liu et al., 2013; Ehlerding et al., 2016). On the other hand, nanoparticles with an overall size larger than $100 \mathrm{~nm}$ may be recognized and removed quickly from the blood stream by the reticuloendothelial system (opsonization by white blood cells, namely macrophages), which will also result in short circulation time (Reimer and Tombach, 1998). Ultrasmall superparamagnetic iron oxide nanoparticles that are smaller than $50 \mathrm{~nm}$ can escape phagocytosis to some extent with a prolonged circulation time (Shen et al., 1993; Wang et al., 2001; Li et al., 2005). An overall size of $15 \mathrm{~nm}$ was found to 

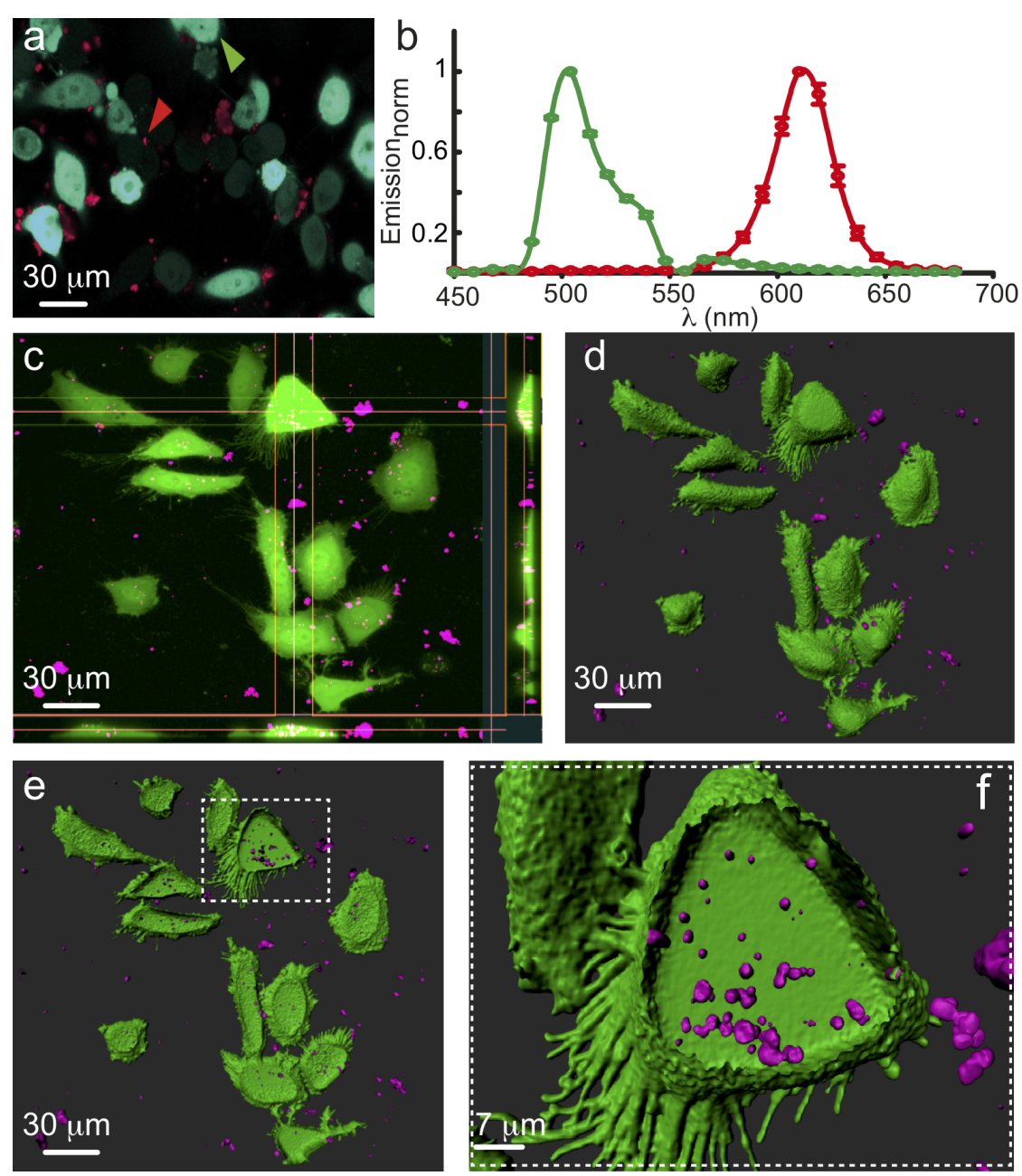

FIGURE 2 | Intracellular Accumulation of Nanoparticles. (a) Spectral micrograph showing HeLa cells transfected with cytosolic GFP (cyan filled cells, green arrowhead) incubated with nanoparticles (NPs; red dots and arrowhead). (b) Emission spectra of GFP (green plot) and NPs (red plot). (c) Projection micrograph (from Z-stack) and orthosteric views showing NPs (magenta) located within individual cells. (d,e) Three dimensional rendering (Imaris) of (c) depicting outer surface (green) of the cells along with the inner volume of the cells containing NPs (magenta). (f) Higher power image showing a single cell filled with at least few dozens of NPs.

result with relatively longer circulation time, affording prolonged MRI signal enhancement effects for over an hour, and collection of high-resolution MR images of blood vessels (Kim et al., 2011). Therefore, longitudinal measurements in the intact animal that depend upon longer circulation time will require unique adjustment and optimization of the overall size of the agent. Together, nanoparticles should not be too small, to prevent rapid central nervous system clearance, and should not be too large, to avoid uptake by macrophages. Thus, the synthetic strategy of choice is critical for appropriate functionality of the magnetofluorescent hybrid structure. In this regard, the yolk-shell strategy may present a convenient design for experiments that require smaller dimensions.

In addition to size, the shape, surface chemistry and aggregation or agglomeration habits of the nanoparticles will also influence the type of internalization process (Albanese and Chan, 2011). In particular, surface coating is found to play an important role in successful delivery of nanoparticles into the intracellular environment (e.g., see Figure 2), and it may additionally offer opportunities for selective labeling of relevant targets. The surface of our yolk-shell magneto-fluorescent nanoparticles is that of the iron oxide shell, rather than silica or polystyrene. This allows implementation of the vast accumulated knowledge that is available in the literature for surface coating and functionalization of iron oxide for the purpose of biological compatibility. Hence, insights on cell internalization and accumulation of the yolk shell magneto fluorescent nanoparticles, which are acquired via utilization of the fluorescent marker, could be directly implemented for similar iron oxide therapeutic nanoparticles. A prominent example relies on distinctive biochemical characteristics of target diseases such as tumor or inflammatory tissue, with surface-functionalization that is designed for improved cell and tissue specific distribution of nanoparticles for localized therapeutic effect, and minimal 
whole-body toxicity (Lee et al., 2015). In addition, along side functionality of the iron oxide nanoparticles as therapeutic hyperthermic agents (Petryk et al., 2013; Hervault and Thanh, 2014), or for controlled drug release through the application of an external magnetic field (Yoo et al., 2011), the QD core could easily be replaced with Au (Shevchenko et al., 2008; Jain et al., 2012) or FePt (Gao et al., 2008) for cancer therapy. Note that such functionalities are hindered or lost entirely if the iron oxide nanoparticles are coated within thick silica or polystyrene shell.

In the course of our work, the CdSe@CdS@hollow- $\mathrm{Fe}_{2} \mathrm{O}_{3}$ yolk-shell magneto-fluorescent nanoparticles (MFNP) were functionalized first with Tiron (disodium 4,5-dihydroxy-1,3benzenedisulfonate) for their incorporation to cells (Korpany et al., 2013). This resulted with stable and well suspended waterdispersed nanoparticles of 14-16 nm hydrodynamic particle size (as determined by dynamic laser scattering analysis). Alternative ligand coatings such as polymer or PEG resulted with drastic increase of the hydrodynamic size, with no added stability. Yet, the intracellular accumulation of Tiron-coated nanoparticles was found to be variable and, at times, limited. Hence, dopamine was examined as an alternative coating (Sherwood et al., 2017). We, and others, envisioned the dopamine-coat to play several critical roles. First, it was expected to increase the solubility of the nanoparticle. Second, it could be recognized by membrane receptors or transporters to promote cell-specific receptormediated endocytosis and internalization of the particles into cells of interest, neurons in particular. Lastly, this coat was also envisioned to play a role in reducing the ability of extracellular proteins to adhere to the nanoparticles, thereby negating their increase in size and clearance by engulfing cells. Though successful to some extent, we have obtained weak LM signals from cells suggesting only moderate cytoplasmic fill. To further enhance endocytosis, we turned to established chemical reagents that are commonly employed for the introduction of foreign material into cells such as DNA. The MFNP were incubated with the transfection reagent lipofectamine, glycerol; a water soluble and hygroscopic lipid, calcium phosphate precipitates and spermidine; a naturally occurring polyamine commonly used in biolistic transfection of neurons. Strikingly, and despite the common features of the reagents, preliminary observations showed that spermidine yielded the best results; with cells displaying detectable intracellular particles with clear evidence of aggregation ( $\sim 1 \mu \mathrm{m}$ in diameter; Figure 2). Notably, external modifications and coatings did not affect the functionality of the encapsulated QDs, as evident by their emission fluorescence spectrum outside or within cells (Figures 2a,b).

The observation of aggregates was surprising, as the nanoparticles consistently remain monomeric in solution in vitro. This therefore suggested to us that the particles are undergoing agglomeration, despite the dopamine coat, and this process is likely mediated by the physiological and/or intracellular conditions. Though unsettling at first, we quickly realized that this process could be beneficial for intracellular trapping of the nanoparticles because as small particles may easily enter the cell, so can they exit it. Deliberate and controlled aggregation may serve for entrapping and accumulating nanometer particles within the cell more readily, in turn providing stronger MRI and fluorescent signals, not to mention increasing the stability of the particles over time due to lower exposure to degradation. This observation also suggests that with further design, nanoparticles could be engineered to undergo activity-dependent aggregation. A relevant and interesting example to explore is aggregation that is induced by the binding of a certain biological moiety that surges in an activity-dependent manner, such as $\mathrm{Ca}^{2+}$-ions (Okada et al., 2018). In this regard, we envision that the coat of the nanoparticles could include small molecules or protein fragments (e.g., Li et al., 1999; Atanasijevic and Jasanoff, 2007; Henig et al., 2011) that bind $\mathrm{Ca}^{2+}$, e.g., calcium-specific aminopolycarboxylic acid such as 1,2-bis(2aminophenoxy)ethane-N,N,N'N'-tetraacetic acid (BAPTA) or Calmodulin, respectively. A plausible scenario would be that following neural activity and sharp rise in cytoplasmic $\mathrm{Ca}^{2+}$ concentration, intracellular nanoparticles would bind $\mathrm{Ca}^{2+}$, thus altering the reactivity of the coating. $\mathrm{Ca}^{2+}$ binding may influence the effective columbic repulsion of the coating, or enhance the affinity and binding to a secondary moiety on the surface of surrounding nanoparticles. Regardless the exact mechanism, it is expected to change the particles' dispersivity in a manner that would result with strong aggregation. This aggregation, once it exceeds a certain size, is expected to result in a detectable MR signal localized to the cell. As $\mathrm{Ca}^{2+}$-binding is reversible, this phenomenon would also be reversible, providing MRI-readout for neuronal activity.

\section{CONCLUSION}

Intracellular magneto-fluorescent contrast agents are of great significance for the development of imaging methodologies for single cell longitudinal measurements in the intact brain. These bi-functional markers enable coupling between high resolution LM with the non-invasive mesoscale imaging capabilities of MRI. Yet the roadmap towards enhancement of MRI signals from select specific cells requires improved understanding of cellular internalization and accumulation mechanisms. In particular, modes of cellular uptake, their efficiency and kinetics, subcellular distribution of contrast agents following uptake, clearance and, notably, toxicity need to be better understood and accurately characterized. These are all influenced, and at times dictated, by the size, shape and surface chemistry of the contrast agent nanoparticles, and should be properly considered when choosing the synthetic strategy. We envision that insights on the correlation between the agents' characteristics and their intracellular distribution would enable manipulation of the latter for improved specificity, and ultimately for functional readout of neuronal activity.

\section{ETHICS STATEMENT}

All of the experiments involving animals were conducted in accordance with the United States Public Health Service's Policy on Humane Care and Use of Laboratory Animals and 
study protocol was approved by the Institutional Animal Care and Use Committee of Technion - Israel Institute of Technology.

\section{AUTHOR CONTRIBUTIONS}

LA, SB, and IK developed the concepts described in this work and wrote the manuscript. SO, SP, and SB carried out the experiments. All authors analyzed the results described in the manuscript and approved the final version of the manuscript. This work is in partial fulfilment for the Degree of Doctor of Philosophy (Ph.D.) for SO.

\section{REFERENCES}

Albanese, A., and Chan, W. C. (2011). Effect of gold nanoparticle aggregation on cell uptake and toxicity. ACS Nano 5, 5478-5489. doi: 10.1021/nn200 7496

Atanasijevic, T., and Jasanoff, A. (2007). Preparation of iron oxide-based calcium sensors for MRI. Nat. Protoc. 2, 2582-2589. doi: 10.1038/nprot.2007.377

Bigall, N. C., Parak, W. J., and Dorfs, D. (2012). Fluorescent, magnetic and plasmonic-hybrid multifunctional colloidal nano objects. Nano Today 7, 282-296. doi: 10.1016/j.nantod.2012.06.007

Boldt, K., Jander, S., Hoppe, K., and Weller, H. (2011). Characterization of the organic ligand shell of semiconductor quantum dots by fluorescence quenching experiments. ACS Nano 5, 8115-8123. doi: 10.1021/nn202748v

Chapman, A. P., Antoniw, P., Spitali, M., West, S., Stephens, S., and King, D. J. (1999). Therapeutic antibody fragments with prolonged in vivo half-lives. Nat. Biotechnol. 17, 780-783. doi: 10.1038/11717

Chen, O., Riedemann, L., Etoc, F., Herrmann, H., Coppey, M., Barch, M., et al. (2014). Magneto-fluorescent core-shell supernanoparticles. Nat. Commun. 5:5093. doi: 10.1038/ncomms6093

Cheng, Y., Morshed, R. A., Auffinger, B., Tobias, A. L., and Lesniak, M. S. (2014). Multifunctional nanoparticles for brain tumor imaging and therapy. Adv. Drug Deliv. Rev. 66, 42-57. doi: 10.1016/j.addr.2013.09.006

Cho, M., Contreras, E. Q., Lee, S. S., Jones, C. J., Jang, W., and Colvin, V. L. (2014). Characterization and optimization of the fluorescence of nanoscale iron oxide/quantum dot complexes. J. Phys. Chem. C 118, 14606-14616. doi: $10.1021 /$ jp502194z

Choi, H. S., Liu, W., Misra, P., Tanaka, E., Zimmer, J. P., Itty Ipe, B., et al. (2007). Renal clearance of quantum dots. Nat. Biotechnol. 25, 1165-1170. doi: 10.1038/ nbt1340

Corr, S. A., Rakovich, Y. P., and Gun'ko, Y. K. (2008). Multifunctional magneticfluorescent nanocomposites for biomedical applications. Nanoscale Res. Lett. 3 , 87-104. doi: 10.1007/s11671-008-9122-8

Dawidczyk, C. M., Russell, L. M., and Searson, P. C. (2014). Nanomedicines for cancer therapy: state-of-the-art and limitations to pre-clinical studies that hinder future developments. Front. Chem. 2:69. doi: 10.3389/fchem.2014. 00069

Di Corato, R., Bigall, N. C., Ragusa, A., Dorfs, D., Genovese, A., Marotta, R., et al. (2011). Multifunctional nanobeads based on quantum dots and magnetic nanoparticles: synthesis and cancer cell targeting and sorting. ACS Nano 5, 1109-1121. doi: 10.1021/nn102761t

Ehlerding, E. B., Chen, F., and Cai, W. (2016). Biodegradable and renal clearable inorganic nanoparticles. Adv. Sci. (Weinh) 3:1500223. doi: 10.1002/advs. 201500223

Erogbogbo, F., Yong, K. T., Hu, R., Law, W. C., Ding, H., Chang, C. W., et al. (2010). Biocompatible magnetofluorescent probes: luminescent silicon quantum dots coupled with superparamagnetic iron(III) oxide. ACS Nano 4, 5131-5138. doi: $10.1021 / \mathrm{nn} 101016 \mathrm{f}$

Fan, H. M., Olivo, M., Shuter, B., Yi, J. B., Bhuvaneswari, R., Tan, H. R., et al. (2010). Quantum dot capped magnetite nanorings as high performance nanoprobe for multiphoton fluorescence and magnetic resonance imaging. J. Am. Chem. Soc. 132, 14803-14811. doi: 10.1021/ja103738t

\section{FUNDING}

This study was supported by the Israel Science Foundation (770/17 and 1096/17), National Institutes of Health (1R01NS091037), the Adelis Foundation and the Allen and Jewel Prince Center for Neurodegenerative Disorders of the Brain. The research was carried out in the framework of the Russell Berrie Nanotechnology Institute (RBNI) and the Lorry I. Lokey Interdisciplinary Center for Life Sciences and Engineering at the Technion. Dr. Pahari expresses his gratitude to Israel Council for Higher Education for the PBC postdoctoral fellowship.

Feld, A., Merkl, J. P., Kloust, H., Flessau, S., Schmidtke, C., Wolter, C., et al. (2015). A universal approach to ultrasmall magneto-fluorescent nanohybrids. Angew. Chem. Int. Ed. Engl. 54, 12468-12471. doi: 10.1002/anie.201503017

Gao, J., Liang, G., Cheung, J. S., Pan, Y., Kuang, Y., Zhao, F., et al. (2008). Multifunctional yolk-shell nanoparticles: a potential mri contrast and anticancer agent. J. Am. Chem. Soc. 130, 11828-11833. doi: 10.1021/ja803920b

Gao, J. H., Gu, H. W., and Xu, B. (2009). Multifunctional magnetic nanoparticles: design, synthesis, and biomedical applications. Acc. Chem. Res. 42, 1097-1107. doi: 10.1021/ar9000026

Gao, Y. (2018). Carbon nano-allotrope/magnetic nanoparticle hybrid nanomaterials as T2 contrast agents for magnetic resonance imaging applications. J. Funct. Biomater. 9:E16. doi: 10.3390/jfb9010016

Geraldes, C. F., and Laurent, S. (2009). Classification and basic properties of contrast agents for magnetic resonance imaging. Contrast Media Mol. Imag. 4, 1-23. doi: 10.1002/cmmi.265

Ghosh, S., Harvey, P., Simon, J. C., and Jasanoff, A. (2018). Probing the brain with molecular fMRI. Curr. Opin. Neurobiol. 50, 201-210. doi: 10.1016/j.conb.2018. 03.009

Gustafson, H. H., Holt-Casper, D., Grainger, D. W., and Ghandehari, H. (2015). Nanoparticle uptake: the phagocyte problem. Nano Today 10, 487-510. doi: 10.1016/j.nantod.2015.06.006

Hachani, R., Birchall, M. A., Lowdell, M. W., Kasparis, G., Tung, L. D., Manshian, B. B., et al. (2017). Assessing cell-nanoparticle interactions by high content imaging of biocompatible iron oxide nanoparticles as potential contrast agents for magnetic resonance imaging. Sci. Rep. 7:7850. doi: 10.1038/s41598-01708092-W

Harris, R. D., Bettis Homan, S., Kodaimati, M., He, C., Nepomnyashchii, A. B., Swenson, N. K., et al. (2016). Electronic processes within quantum dot-molecule complexes. Chem. Rev. 116, 12865-12919. doi: 10.1021/acs.chemrev.6b 00102

He, C., Hu, Y., Yin, L., Tang, C., and Yin, C. (2010). Effects of particle size and surface charge on cellular uptake and biodistribution of polymeric nanoparticles. Biomaterials 31, 3657-3666. doi: 10.1016/j.biomaterials.2010.01. 065

Henig, J., Mamedov, I., Fouskova, P., Toth, E., Logothetis, N. K., Angelovski, G., et al. (2011). Influence of calcium-induced aggregation on the sensitivity of aminobis(methylenephosphonate)-containing potential MRI contrast agents. Inorg. Chem. 50, 6472-6481. doi: 10.1021/ic1024235

Hervault, A., and Thanh, N. T. (2014). Magnetic nanoparticle-based therapeutic agents for thermo-chemotherapy treatment of cancer. Nanoscale 6, 11553-11573. doi: 10.1039/C4NR03482A

Huang, J., Bu, L., Xie, J., Chen, K., Cheng, Z., Li, X., et al. (2010). Effects of nanoparticle size on cellular uptake and liver MRI with polyvinylpyrrolidonecoated iron oxide nanoparticles. ACS Nano 4, 7151-7160. doi: 10.1021/ nn $101643 \mathrm{u}$

Insin, N., Tracy, J. B., Lee, H., Zimmer, J. P., Westervelt, R. M., and Bawendi, M. G. (2008). Incorporation of iron oxide nanoparticles and quantum dots into silica microspheres. ACS Nano 2, 197-202. doi: 10.1021/nn700344x

Jain, S., Hirst, D. G., and O'sullivan, J. M. (2012). Gold nanoparticles as novel agents for cancer therapy. Br. J. Radiol. 85, 101-113. doi: 10.1259/bjr/5944 8833 
Kas, R., Sevinc, E., Topal, U., and Acar, H. Y. (2010). A universal method for the preparation of magnetic and luminescent hybrid nanoparticles. J. Phys. Chem. C 114, 7758-7766. doi: 10.1021/jp100312e

Kievit, F. M., and Zhang, M. (2011). Surface engineering of iron oxide nanoparticles for targeted cancer therapy. Acc. Chem. Res. 44, 853-862. doi: 10.1021/ar2000277

Kim, B. H., Lee, N., Kim, H., An, K., Park, Y. I., Choi, Y., et al. (2011). Large-scale synthesis of uniform and extremely small-sized iron oxide nanoparticles for high-resolution T1 magnetic resonance imaging contrast agents. J. Am. Chem. Soc. 133, 12624-12631. doi: 10.1021/ja203340u

Kim, B. S., and Taton, T. A. (2007). Multicomponent nanoparticles via selfassembly with cross-linked block copolymer surfactants. Langmuir 23, 21982202. doi: 10.1021/la062692w

Kim, J., Kim, H. S., Lee, N., Kim, T., Kim, H., Yu, T., et al. (2008). Multifunctional uniform nanoparticles composed of a magnetite nanocrystal core and a mesoporous silica shell for magnetic resonance and fluorescence imaging and for drug delivery. Angew. Chem. Int. Edn. 47, 8438-8441. doi: 10.1002/anie. 200802469

Kim, J., Lee, J. E., Lee, J., Yu, J. H., Kim, B. C., An, K., et al. (2006). Magnetic fluorescent delivery vehicle using uniform mesoporous silica spheres embedded with monodisperse magnetic and semiconductor nanocrystals. J. Am. Chem. Soc. 128, 688-689. doi: 10.1021/ja0565875

Kim, S., Lim, Y. T., Soltesz, E. G., De Grand, A. M., Lee, J., Nakayama, A., et al. (2004). Near-infrared fluorescent type II quantum dots for sentinel lymph node mapping. Nat. Biotechnol. 22, 93-97. doi: 10.1038/nbt920

Kim, S. G., and Ogawa, S. (2012). Biophysical and physiological origins of blood oxygenation level-dependent fMRI signals. J. Cereb. Blood Flow Metab. 32, 1188-1206. doi: 10.1038/jcbfm.2012.23

Korpany, K. V., Habib, F., Murugesu, M., and Blum, A. S. (2013). Stable watersoluble iron oxide nanoparticles using Tiron. Mater. Chem. Phys. 138, 29-37. doi: 10.1016/j.matchemphys.2012.10.015

Lee, N., Yoo, D., Ling, D., Cho, M. H., Hyeon, T., and Cheon, J. (2015). Iron oxide based nanoparticles for multimodal imaging and magnetoresponsive therapy. Chem. Rev. 115, 10637-10689. doi: 10.1021/acs.chemrev.5b00112

Li, C. (2014). A targeted approach to cancer imaging and therapy. Nat. Mater. 13, 110-115. doi: 10.1038/nmat3877

Li, W., Tutton, S., Vu, A. T., Pierchala, L., Li, B. S., Lewis, J. M., et al. (2005). First-pass contrast-enhanced magnetic resonance angiography in humans using ferumoxytol, a novel ultrasmall superparamagnetic iron oxide (USPIO)-based blood pool agent. J. Magn. Reson Imaging 21, 46-52. doi: 10.1002/jmri.20235

Li, W.-H., Fraser, S. E., and Meade, T. J. (1999). A calcium-sensitive magnetic resonance imaging contrast agent. J. Am. Chem. Soc. 121, 1413-1414. doi: 10.1021/ja9837021

Lim, E. K., Kim, T., Paik, S., Haam, S., Huh, Y. M., and Lee, K. (2015). Nanomaterials for theranostics: recent advances and future challenges. Chem. Rev. 115, 327-394. doi: 10.1021/cr300213b

Liu, J. B., Yu, M. X., Zhou, C., and Zheng, J. (2013). Renal clearable inorganic nanoparticles: a new frontier of bionanotechnology. Mater. Today 16, 477-486. doi: 10.1016/j.mattod.2013.11.003

Logothetis, N. K. (2008). What we can do and what we cannot do with fMRI. Nature 453, 869-878. doi: 10.1038/nature06976

Medintz, I. L., Uyeda, H. T., Goldman, E. R., and Mattoussi, H. (2005). Quantum dot bioconjugates for imaging, labelling and sensing. Nat. Mater. 4, 435-446. doi: $10.1038 /$ nmat 1390

Mukherjee, A., Davis, H. C., Ramesh, P., Lu, G. J., and Shapiro, M. G. (2017). Biomolecular MRI reporters: evolution of new mechanisms. Prog. Nucl. Magn. Reson Spectrosc. 10, 32-42. doi: 10.1016/j.pnmrs.2017.05.002

Muthu, M. S., Leong, D. T., Mei, L., and Feng, S. S. (2014). Nanotheranostics Application and further development of nanomedicine strategies for advanced theranostics. Theranostics 4, 660-677. doi: 10.7150/thno.8698

Oh, N., and Park, J. H. (2014). Endocytosis and exocytosis of nanoparticles in mammalian cells. Int. J. Nanomed. 9(Suppl. 1), 51-63.

Okada, S., Bartelle, B. B., Li, N., Breton-Provencher, V., Lee, J. J., Rodriguez, E., et al. (2018). Calcium-dependent molecular fMRI using a magnetic nanosensor. Nat. Nanotechnol. 13, 473-477. doi: 10.1038/s41565-0180092-4

Olmsted, S. S., Padgett, J. L., Yudin, A. I., Whaley, K. J., Moench, T. R., and Cone, R. A. (2001). Diffusion of macromolecules and virus-like particles in human cervical mucus. Biophys. J. 81, 1930-1937. doi: 10.1016/S0006-3495(01)75 844-4

Ortgies, D. H., De La Cueva, L., Del Rosal, B., Sanz-Rodriguez, F., Fernandez, N., Iglesias-De, et al. (2016). In vivo deep tissue fluorescence and magnetic imaging employing hybrid nanostructures. ACS Appl. Mater. Interfaces 8, 1406-1414. doi: 10.1021/acsami.5b10617

Ow, H., Larson, D. R., Srivastava, M., Baird, B. A., Webb, W. W., and Wiesner, U. (2005). Bright and stable core-shell fluorescent silica nanoparticles. Nano Lett. 5, 113-117. doi: 10.1021/nl0482478

Pahari, S. K., Olszakier, S., Kahn, I., and Amirav, L. (2018). Magnetofluorescent yolk-shell nanoparticles. Chem. Mater. 30, 775-780. doi: 10.1021/ acs.chemmater.7b04253

Park, J. H., von Maltzahn, G., Ruoslahti, E., Bhatia, S. N., Sailor, M. J., and Micellar, M. (2008). Hybrid nanoparticles for simultaneous magnetofluorescent imaging and drug delivery. Angew. Chem. Int. Ed. Engl. 47, 7284-7288. doi: 10.1002/anie.200801810

Petryk, A. A., Giustini, A. J., Gottesman, R. E., Kaufman, P. A., and Hoopes, P. J. (2013). Magnetic nanoparticle hyperthermia enhancement of cisplatin chemotherapy cancer treatment. Int. J. Hyperth. 29, 845-851. doi: 10.3109/ 02656736.2013.825014

Piao, Y., Burns, A., Kim, J., Wiesner, U., and Hyeon, T. (2008). Fabrication of silica-based nanostructured particle systems for nanomedicine applications. Adv. Funct. Mater. 18, 3745-3758. doi: 10.1002/adfm.200800731

Qiao, R., Jia, Q., Huwel, S., Xia, R., Liu, T., Gao, F., et al. (2012). Receptor-mediated delivery of magnetic nanoparticles across the blood-brain barrier. ACS Nano 6, 3304-3310. doi: 10.1021/nn300240p

Qin, Z., and Bischof, J. C. (2012). Thermophysical and biological responses of gold nanoparticle laser heating. Chem. Soc. Rev. 41, 1191-1217. doi: 10.1039/ C1CS15184C

Reimer, P., and Tombach, B. (1998). Hepatic MRI with SPIO: detection and characterization of focal liver lesions. Eur. Radiol. 8, 1198-1204. doi: 10.1007/ s003300050535

Roullier, V., Grasset, F., Boulmedais, F., Artzner, F., Cador, O., R., Marchi-Artzner, $\mathrm{V}$ et al. (2008). Small bioactivated magnetic quantum dot micelles. Chem. Mater. 20, 6657-6665. doi: 10.1021/cm801423r

Sailor, M. J., and Park, J. H. (2012). Hybrid nanoparticles for detection and treatment of cancer. Adv. Mater. 24, 3779-3802. doi: 10.1002/adma.201200653

Saka, S. K., Honigmann, A., Eggeling, C., Hell, S. W., Lang, T., and Rizzoli, S. O. (2014). Multi-protein assemblies underlie the mesoscale organization of the plasma membrane. Nat. Commun. 5:4509. doi: 10.1038/ncomms5509

Sanna, V., Pala, N., and Sechi, M. (2014). Targeted therapy using nanotechnology: focus on cancer. Int. J. Nanomed. 9, 467-483.

Selvan, S. T., Patra, P. K., Ang, C. Y., and Ying, J. Y. (2007). Synthesis of silicacoated semiconductor and magnetic quantum dots and their use in the imaging of live cells. Angew. Chem. Int. Ed Engl. 46, 2448-2452. doi: 10.1002/anie. 200604245

Shang, L., Nienhaus, K., and Nienhaus, G. U. (2014). Engineered nanoparticles interacting with cells: size matters. J. Nanobiotechnol. 12:5. doi: 10.1186/14773155-12-5

Shen, T., Weissleder, R., Papisov, M., Bogdanov, A. Jr., and Brady, T. J. (1993). Monocrystalline iron oxide nanocompounds (MION): physicochemical properties. Magn. Reson. Med. 29, 599-604. doi: 10.1002/mrm.19102 90504

Shen, Z., Wu, A., and Chen, X. (2017). Iron oxide nanoparticle based contrast agents for magnetic resonance imaging. Mol. Pharm. 14, 1352-1364. doi: 10.1021/acs.molpharmaceut.6b00839

Sherwood, S., Xu, Y., Lovas, K., Qin, Y., and Bao, Y. (2017). Surface functionalization of dopamine coated iron oxide nanoparticles for various surface functionalities. J. Magn. Magn. Mater. 427, 220-224. doi: 10.1016/j. jmmm.2016.10.039

Shevchenko, E. V., Bodnarchuk, M. I., Kovalenko, M. V., Talapin, D. V., Smith, R. K., Aloni, S., et al. (2008). Gold/iron oxide core/hollow-shell nanoparticles. Adv. Mater. 20, 4323-4329. doi: 10.1002/adma.200702994

Shibu, E. S., Ono, K., Sugino, S., Nishioka, A., Yasuda, A., Shigeri, Y., et al. (2013). Photouncaging nanoparticles for MRI and fluorescence imaging in vitro and in vivo. ACS Nano 7, 9851-9859. doi: 10.1021/nn4043699

Silva, A. J. (2017). Miniaturized two-photon microscope: seeing clearer and deeper into the brain. Light: Sci. Appl. 6:e17104. doi: 10.1038/lsa.2017.104 
Sitbon, G., Bouccara, S., Tasso, M., Francois, A., Bezdetnaya, L., Marchal, F., et al. (2014). Multimodal Mn-doped I-III-VI quantum dots for near infrared fluorescence and magnetic resonance imaging: from synthesis to in vivo application. Nanoscale 6, 9264-9272. doi: 10.1039/C4NR02239D

Suh, W. H., Suh, Y. H., and Stucky, G. D. (2009). Multifunctional nanosystems at the interface of physical and life sciences. Nano Today 4, 27-36. doi: 10.1016/j. nantod.2008.10.013

Uchiyama, M. K., Toma, S. H., Rodrigues, S. F., Shimada, A. L., Loiola, R. A., Cervantes Rodriguez, H. J., et al. (2015). Ultrasmall cationic superparamagnetic iron oxide nanoparticles as nontoxic and efficient MRI contrast agent and magnetic-targeting tool. Int. J. Nanomed. 10, 4731-4746.

Ugurbil, K. (2016). What is feasible with imaging human brain function and connectivity using functional magnetic resonance imaging. Philos. Trans. R. Soc. Lond. B Biol. Sci. 371:20150361. doi: 10.1098/rstb.2015.0361

Verma, A., and Stellacci, F. (2010). Effect of surface properties on nanoparticle-cell interactions. Small 6, 12-21. doi: 10.1002/smll.200901158

Wang, Y. X., Hussain, S. M., and Krestin, G. P. (2001). Superparamagnetic iron oxide contrast agents: physicochemical characteristics and applications in MR imaging. Eur. Radiol. 11, 2319-2331. doi: 10.1007/s003300100908

Wysocki, L. M., and Lavis, L. D. (2011). Advances in the chemistry of small molecule fluorescent probes. Curr. Opin. Chem. Biol. 15, 752-759. doi: 10.1016/ j.cbpa.2011.10.013
Yoo, D., Lee, J. H., Shin, T. H., and Cheon, J. (2011). Theranostic magnetic nanoparticles. Acc. Chem. Res. 44, 863-874. doi: 10.1021/ar200085c

Zhang, S., Gao, H., and Bao, G. (2015). Physical principles of nanoparticle cellular endocytosis. ACS Nano 9, 8655-8671. doi: 10.1021/acsnano.5b03184

Zheng, Q., Jockusch, S., Zhou, Z., and Blanchard, S. C. (2014). The contribution of reactive oxygen species to the photobleaching of organic fluorophores. Photochem. Photobiol. 90, 448-454. doi: 10.1111/php.12204

Zong, W., Wu, R., Li, M., Hu, Y., Li, Y., Li, J., et al. (2017). Fast high-resolution miniature two-photon microscopy for brain imaging in freely behaving mice. Nat. Methods 14, 713-719. doi: 10.1038/nmeth.4305

Conflict of Interest Statement: The authors declare that the research was conducted in the absence of any commercial or financial relationships that could be construed as a potential conflict of interest.

Copyright $\odot 2019$ Amirav, Berlin, Olszakier, Pahari and Kahn. This is an open-access article distributed under the terms of the Creative Commons Attribution License (CC BY). The use, distribution or reproduction in other forums is permitted, provided the original author(s) and the copyright owner(s) are credited and that the original publication in this journal is cited, in accordance with accepted academic practice. No use, distribution or reproduction is permitted which does not comply with these terms. 